\title{
Analysis of the light dependent resistor configuration for line tracking robot application
}

\begin{abstract}
Modern days, a robot can be designed based on human needs. In particular, the line tracking robot is a classic introductory robot design and needs minimal amount of material and cost. The aim of this project is to construct a robot that has capability to follow the white line placed on a horizontal smooth surface lighted by LED and the low cost light dependant resistor as the sensor. Rigorous analysis has been applied to determine the optimal configuration for the sensor on the mobile robot. Our findings show that angle of LDR of $30^{\circ}$, the distance between LDR and LED of $2.5 \mathrm{~cm}$ and height of $2 \mathrm{~cm}$ is the best configuration. The line tracking robot will detect the light intensity that is rebounded from the white color path.
\end{abstract}

Keyword: Light dependant resistor (LDR); Low cost sensor; PIF16F887; Stepper motor 